\title{
Exploring the relationship between maternal iron status and offspring's blood pressure and adiposity: a Mendelian randomization study
}

This article was published in the following Dove Press journal:

Clinical Epidemiology

8 August 2012

Number of times this article has been viewed

\section{Nisreen A Alwan' \\ Debbie A Lawlor ${ }^{2}$ \\ Harry J McArdle ${ }^{3}$ \\ Darren C Greenwood ${ }^{4}$ \\ Janet E Cade'}

'Nutritional Epidemiology Group, School of Food Science and Nutrition, University of Leeds, Leeds, UK; ${ }^{2}$ MRC Centre for Causal Analyses in Translational Research, University of Bristol, Bristol; ${ }^{3}$ Rowett Institute of Nutrition and Health, University of Aberdeen, Aberdeen; ${ }^{4}$ Biostatistics Unit, Leeds Institute for Health, Genetics and Therapeutics, University of Leeds, Leeds, UK
Correspondence: Nisreen A Alwan Nutritional Epidemiology Group, University of Leeds, School of Food Science and Nutrition, Leeds LS2 9JT, UK

Tel +44 II3 3436990

Fax +44 II 33436587

Emailn.alwan@leeds.ac.uk
Background: Iron deficiency is the most common micronutrient deficiency worldwide. Experimental animal studies suggest that mothers deficient in iron during pregnancy are more likely to have offspring who become obese with high blood pressure. $C 282 Y$ mutation carriers are more likely to have higher iron stores.

Methods: We undertook an instrumental variable (IV) analysis, using maternal $C 282 \mathrm{Y}$ as an indicator for the mother's iron status, to examine its association with offspring blood pressure (BP), waist circumference (WC), and body mass index (BMI), and compared the results to that of ordinary least squares (OLS) regression. Offspring of a sub-cohort of mothers from the UK Women's Cohort Study (UKWCS) were recruited in 2009-2010 $(n=348$, mean age $=41$ years). Their blood pressure, height, and weight were measured at their local general medical practice, and they were asked to self-measure their waist circumference. About half were offspring of $C 282 Y$ carriers. Maternal ferritin was used as a biomarker of maternal iron status.

Results: Maternal $C 282 Y$ was strongly associated with maternal ferritin (mean difference per allele $=84 \mathrm{~g} / \mathrm{L}, 95 \%$ confidence interval: $31-137, P=0.002)$. Using IV analyses, maternal ferritin was not linked to offspring's BP, BMI, or WC. The first stage $F$-statistic for the strength of the instrument was 10 (Kleibergen-Paap $r k$ LM $P=0.009$ ). Maternal ferritin was linked to offspring diastolic BP, WC, and BMI in univariable, but not in multivariable OLS analysis. There was no difference between the OLS and the IV models coefficients for any of the outcomes considered.

Conclusion: We found no association between maternal iron status and adult offspring's BP and adiposity using both multivariable OLS and IV modeling. To our knowledge, this is the first study examining this relationship. Further exploration in larger studies that have genetic variation assessed in both mother and offspring should be considered.

Keywords: iron, pregnancy, developmental origins, Mendelian randomization

\section{Introduction}

Iron deficiency is the leading single nutrient deficiency in the world. Fifty percent of pregnant women in developing countries are anemic mostly due to iron deficiency. It is the only micronutrient deficiency which is also significantly prevalent in high income countries. ${ }^{1}$ Maternal iron deficiency during pregnancy in animal experiments results in the development of obesity and hypertension in offspring despite the offspring having normal iron levels throughout postnatal life. ${ }^{2-5}$ Iron deficiency-induced changes in maternal metabolism may have downstream effects on placental structure, endocrine and transport placental functions, nutrient interactions, and fetal organ development such as reduced kidney nephron number. ${ }^{6}$ However, association studies of specific 
nutrient effects in humans are difficult because of the likelihood of important confounding by other nutrients or dietary characteristics, as well as other lifestyle factors such as smoking and alcohol consumption. ${ }^{7}$

The use of the random assortment of genes from parents to offspring provides one method for assessing the causal nature of some environmental exposures. This approach, known as Mendelian randomization (MR), uses genetic variants as instrumental variables (IV) for the environmental exposures of interest. ${ }^{8}$ Genetic variants are not influenced by the many lifestyle and environmental characteristics that risk factors, such as iron deficiency, are associated with, ${ }^{9}$ and since they are allocated at conception cannot be influenced by the later occurrence of disease processes or treatment either. Therefore, associations derived from MR analysis are less likely to be affected by confounding and reverse causality that can bias established multivariable approaches to epidemiological association studies. ${ }^{10}$

Women who carry a $C 282 Y$ mutation in the $H F E$ gene are more likely, in the homozygous state, to suffer from hemochromatosis, resulting in iron overload in the liver. ${ }^{11,12}$ About $12 \%-20 \%$ of Northern Europeans are heterozygotes for this mutation. ${ }^{11} H F E$ gene mutation carriers are usually asymptomatic, however there is evidence that $C 282 \mathrm{Y}$ heterozygotes have higher total iron stores reflected by higher transferrin saturation levels, ${ }^{13-16}$ and higher total body iron stores reflected by lower transferrin receptor:serum ferritin ratios. ${ }^{13} \mathrm{C} 282 Y$ homozygotes have significantly higher ferritin, transferrin saturation and serum iron levels compared to wild type individuals. ${ }^{14}$ In this study, we have used serum ferritin as the environmental measure of the exposure of interest; maternal iron status, as it is considered the most useful laboratory measure of iron status in the absence of inflammation. ${ }^{17}$

IV analysis uses the proportion of the variation in maternal iron status that is explained by $C 282 Y$ to provide an unconfounded estimate of the relationship with offspring outcomes. We hypothesize that women who carry the $C 282 Y$ mutation are at lower risk of iron deficiency during pregnancy, and therefore their offspring will have on average lower blood pressure and adiposity (as indicated by body mass index [BMI] and waist circumference [WC]). The aim of this study was to examine the relationship between maternal iron status and offspring BP and adiposity measures in adulthood, using both ordinary multivariable regression and IV regression methods.

\section{Methods}

The UK Women's Cohort Study (UKWCS), which includes 35,372 women, aged 35-69 years at recruitment across
England, Wales, and Scotland, was established in 1994. The cohort is described in detail elsewhere. ${ }^{18}$ Approximately 15,000 women from UKWCS were contacted between 1999 and 2002 and asked to provide cheek cell samples for DNA extraction (Phase II data collection). All subjects who were found to be homozygous or heterozygous for the C282Y gene mutation were also asked to provide a blood sample, to confirm the result from the cheek cell DNA and to measure markers of iron status including serum ferritin. The method of analysis to detect the mutation is described in detail elsewhere. ${ }^{14}$ In addition, 3000 women were randomly selected to act as a control group. ${ }^{14}$ We have re-contacted 1416 mothers (identified as reporting having at least one live child in the first UKWCS questionnaire) between 2008 and 2010, of whom 716 were $C 282 Y$ allele carriers ( $a a$ or $a g$ ), and 700 were wild type HFE genotype $(g g)$. The non-exposed in this sample were randomly selected from the pool of women with one or more children who were tested and found to have a wild type genotype. The total number of offspring for women contacted in this study was 3376, of whom 1686 were children to $C 282 Y$ carrier mothers and 1690 to wild type mothers.

The mothers were asked to pass on the study information sheet and consent forms to their offspring. The offspring who consented to take part in the study were asked to self-measure their WC following specific instructions using a tape measure supplied in the study pack. Two measurements for each (in inches or centimeters) were provided and their mean used in all analyses. Height, weight, and BP were measured at each participant's general practice. A standard operating procedure for the measurement of height, weight, and BP sheet was attached to the GP/practice nurse's letter and practice staff were asked to follow it when taking the measurements. We asked for two BP measurements at least 1 minute apart. If the first and second measurement differed by more than $5 \mathrm{mmHg}$ for either the systolic blood pressure (SBP) or diastolic blood pressure (DBP), a third measurement was requested at least 1 minute after the second measurement. The mean of the two or three readings was used in all analyses. Each participant was also asked to fill in a lifestyle questionnaire.

\section{Statistical analysis}

All analyses were performed using Stata (v 11.0; Stata Corporation, College Station, TX). The relationship of interest was examined using both ordinary least squares (OLS) regression and instrumental variable (IV) regression modeling using the 'ivreg2' command in Stata. We adjusted for clustering of siblings (where there was more than one 
sibling per mother included in the study) using the 'robust cluster' command in Stata which computes a cluster-robust standard error of the difference in both the OLS and the IV analyses.

We used two-stage least square (2sls) as a method of estimation for IV regression, with maternal $C 282 Y$ genotype $(a a, a g$ or $g g$ ) as the instrument, under an additive model, and maternal ferritin as the modifiable risk factor of interest. We included SBP, DBP, WC, and BMI, as outcomes. In the first stage of the 2SLS model, the regression of maternal ferritin on $C 282 Y$ (instrument) is fitted. In the second stage, the outcome is regressed on the predicted values of maternal Fn, the coefficient of which is the estimate of the causal effect. The standard errors of the second stage parameters are appropriately corrected to account for the uncertainty in the predicted values of the exposure from the first stage. The first stage $F$-statistic is reported to provide an indicator of the instrument strength. Values of 10 and over are taken to be sufficient to exclude weak identification using the specified instrument (maternal C282Y). ${ }^{19}$ The weak identification $F$-statistic we used for the IV regression in the case of specification of 'robust cluster' in the model is based on the Kleibergen-Paap rk statistic, the null hypothesis being that the estimator is weakly identified by the instrument. ${ }^{20}$ Rejecting the null hypothesis indicates that the instrument has sufficient strength. ${ }^{20}$

Child age, maternal age (at Phase II of the UKWCS), and child gender were considered confounders and adjusted for in the multivariable OLS models. In addition, maternal BMI (at Phase II) and maternal social class (as classified according to the three category National Statistics Socio-economic Classification) were considered confounders and adjusted for in the multivariable OLS models with offspring BMI and WC as outcomes. Other potential confounding factors such as mother's vegetarian status was tested for association with the exposure and offspring's blood pressure and adiposity, and were included in the multivariable OLS model if they showed a statistically significant relationship with both.

The two modeling methods used (OLS and IV regression) are examining the same association of maternal iron status and offspring adiposity and blood pressure outcomes, using the difference in SBP, DBP, WC, and BMI per $10 \mu \mathrm{g} / \mathrm{L}$ greater maternal serum ferritin. None of the models are testing the direct link between the maternal C282Y genotype and offspring outcomes. We used 10,000 bootstrapped replications to estimate the standard errors of the differences between the IV and the multivariable OLS estimates and then calculated $P$-values from these standard errors based on normal approximation for the sampling distribution of the mean differences.

This study was conducted according to the guidelines laid down in the Declaration of Helsinki and all procedures involving human subjects were approved by the NHS North West Research Ethical Committee (REC reference number 07/H1010/68). Written informed consent was obtained from all subjects.

\section{Results}

The characteristics and measurements of the study participants are described in Tables 1 and 2. Five hundred and seventeen of the original 3376 offspring consented to take part (17\%), and 348 offspring of 277 mothers completed the study ( $10 \%$ of the invited sample, $61 \%$ of those who consented).

Table I Offpsring's characteristics

\begin{tabular}{|c|c|c|c|}
\hline & Number & Percentage & $95 \% \mathrm{Cl}$ \\
\hline \multicolumn{4}{|l|}{ Body mass index $(n=335)$} \\
\hline$<25$ & 214 & 63.9 & $58.5-69.0$ \\
\hline $25-29$ & 88 & 26.3 & $21.6-31.3$ \\
\hline$\geq 30$ & 33 & 9.9 & $6.9-13.6$ \\
\hline \multicolumn{4}{|l|}{ Smoking $(n=340)$} \\
\hline Current smoker & 33 & 9.7 & $6.8-1.3$ \\
\hline Ex-smoker & 106 & 31.2 & $26.3-36.4$ \\
\hline Never smoked & 201 & 59.1 & $53.7-64.4$ \\
\hline \multicolumn{4}{|l|}{ Alcohol intake $(n=339)$} \\
\hline Regular & 207 & 61.1 & $55.6-66.3$ \\
\hline Once/week & 46 & 13.6 & $10.1-17.7$ \\
\hline Occasional/never & 86 & 25.4 & $20.8-30.4$ \\
\hline \multicolumn{4}{|l|}{ Marital status $(n=333)$} \\
\hline Married & 105 & 55.6 & $50.0-60.9$ \\
\hline Single & 115 & 34.5 & $29.4-39.9$ \\
\hline Divorced/separated/widowed & 33 & 9.9 & $6.9-13.6$ \\
\hline Vegetarian $(n=332)$ & 44 & 13.3 & $10.0-17.4$ \\
\hline Hypertension & 21 & 6.2 & $3.9-9.3$ \\
\hline \multicolumn{4}{|l|}{ (self-reported, $n=340$ ) } \\
\hline $\mathrm{SBP}>140 \mathrm{mmHg}(\mathrm{n}=336)$ & 13 & 3.9 & $2.1-6.5$ \\
\hline $\mathrm{DBP}>90 \mathrm{mmHg}(\mathrm{n}=336)$ & 7 & 2.1 & $0.8-4.2$ \\
\hline \multicolumn{4}{|l|}{ Any exercise during the week } \\
\hline Vigorous $(n=332)$ & 197 & 59.5 & $53.8-64.7$ \\
\hline Moderate $(n=337)$ & 259 & 76.6 & $72.0-81.3$ \\
\hline Light $(n=339)$ & 315 & 92.9 & $89.6-95.4$ \\
\hline \multirow{2}{*}{\multicolumn{4}{|c|}{$\begin{array}{l}\text { Self-reported occupation } \\
(\mathrm{n}=286)\end{array}$}} \\
\hline & & & \\
\hline Professional/senior managers & 165 & 57.7 & $51.7-63.5$ \\
\hline Intermediate/technical & 54 & 18.9 & $|4.5-24|$. \\
\hline Routine/semi-routine manual & 15 & 5.2 & $3.0-8.5$ \\
\hline Self-employed & 41 & 14.3 & $10.5-18.9$ \\
\hline Student & 9 & 3.2 & I.4-5.9 \\
\hline Unemployed & 2 & 0.7 & $0.08-2.5$ \\
\hline $\begin{array}{l}\text { Self-reported low birthweight } \\
(<2500 \mathrm{~g})(\mathrm{n}=230)\end{array}$ & 15 & 6.5 & $3.7-10.5$ \\
\hline
\end{tabular}

Abbreviations: $\mathrm{Cl}$, confidence interval; DBP, diastolic blood pressure; SBP, systolic blood pressure. 
Table 2 Offspring's measurements

\begin{tabular}{lllll}
\hline & Mean & SD & $95 \%$ CI & Median \\
\hline Height $(n=335)$ & 169.3 & 9.8 & $168.3-170.4$ & 168 \\
F & 165.2 & 7.0 & $164.3-166.1$ & 165 \\
M & 179.7 & 8.1 & $178.1-181.4$ & 179 \\
Weight $(n=338)$ & 70.0 & 14.3 & $68.5-71.5$ & 66.7 \\
F & 65.5 & 12.1 & $78.2-83.5$ & 64.0 \\
M & 80.9 & 13.3 & $64.0-67.1$ & 79.8 \\
BMI $(n=335)$ & 24.3 & 4.2 & $23.9-24.8$ & 23.6 \\
F & 24.0 & 4.3 & $23.5-24.6$ & 23.3 \\
M & 25.0 & 3.9 & $24.2-25.8$ & 24.6 \\
SBP $(n=336)$ & 116.5 & 13.6 & $115.0-117.9$ & 116.0 \\
F & 113.6 & 12.6 & $112.0-115.2$ & 113.0 \\
M & 123.5 & 13.5 & $120.8-126.2$ & 122.5 \\
DBP $(n=336)$ & 72.7 & 9.2 & $71.8-73.7$ & 72.0 \\
F & 71.4 & 8.7 & $70.3-72.5$ & 71.0 \\
M & 76.0 & 9.7 & $74.0-77.9$ & 76.0 \\
Waist circumference $(n=34 I)$ & 83.2 & 12.4 & $81.9-84.5$ & 81.3 \\
F & 80.5 & 11.3 & $79.1-81.9$ & 78.3 \\
M & 89.7 & 12.7 & $87.2-92.2$ & 89.5 \\
Hip circumference $(n=342)$ & 98.9 & 10.1 & $97.9-100.0$ & 98.5 \\
F & 98.9 & 10.1 & $97.6-100.2$ & 97.5 \\
M & 99.0 & 10.1 & $97.0-101.0$ & 100 \\
Waist/hip ratio $(n=341)$ & 0.84 & 0.09 & $0.83-0.85$ & 0.83 \\
F & 0.81 & 0.07 & $0.81-0.82$ & 0.81 \\
M & 0.91 & 0.11 & $0.89-0.93$ & 0.91 \\
\hline A6 & & &
\end{tabular}

Abbreviations: $\mathrm{BMI}$, body mass index; $\mathrm{Cl}$, confidence interval; DBP, diastolic blood pressure; SBP, systolic blood pressure, $F$, female; $M$, male.

One hundred and seventy (49\%) were children of $C 282 Y$ mutation genotype mothers, of whom 12 were children of 11 homozygotes mothers ( $a a)$, and 158 were children of heterozygous mothers $(\mathrm{ag})$. Two hundred and forty-seven (71\%) were females and 101 (29\%) were males. The mean age was 41 years for women ( $95 \%$ confidence intervals [CI]: 40-41) and 40 years (95\% CI: 38-42) for men. All participants who reported ethnicity information $(n=335)$ described their ethnic group as white. Two hundred and twelve offspring had no other siblings, 117 had one sibling included in the study, 15 had two siblings, and four had three siblings included. Four percent $(\mathrm{n}=13)$ had systolic, and $2 \%$ $(n=7)$ had diastolic hypertension, according to the WHO cutoff points of 140/90 mmHg. ${ }^{21}$ Figure 1 shows the average offspring BP, WC, and BMI for each maternal genotype.

Maternal serum ferritin was associated with maternal age, offspring age, maternal and offspring vegetarian status, but there was no statistical evidence that maternal $C 282 \mathrm{Y}$ genotype was associated with any of these factors (Table 3). Maternal $C 282 Y$ was strongly associated with maternal Fn: mean difference per each additional allele $=84 \mathrm{~g} / \mathrm{L}(95 \%$ CI: $31-137, P=0.002)$. In univariable analysis, maternal ferritin was linked to offspring's DBP $(0.1 \mathrm{mmHg}$ for every $10 \mu \mathrm{g} / \mathrm{L}$ increase in maternal ferritin, 95\% CI: $0.03-0.3$,
$P=0.02)$, offspring WC $(0.2 \mathrm{~cm}$ for every $10 \mu \mathrm{g} / \mathrm{L}$ increase in maternal ferritin, $95 \% \mathrm{CI}$ : $0.06-0.3, P=0.006$ ), and offspring's BMI $\left(0.05 \mathrm{~kg} / \mathrm{m}^{2}\right.$ for every $10 \mu \mathrm{g} / \mathrm{L}$ increase in maternal ferritin, $95 \% \mathrm{CI}$ : $0.002-0.1, P=0.04)$. There were no differences in mean offspring BP, WC, and BMI between $C 282 Y$ carriers $(a a, a g)$ and wild type mothers $(g g)$ (results not shown). Maternal vegetarian status was significantly linked to both maternal ferritin and offspring DBP, and therefore was additionally entered in the multivariable OLS model with DBP as the outcome.

Table 4 shows the results of associations of maternal Fn with each offspring outcome from both the confounderadjusted multivariable analyses and the IV analyses using maternal $C 282 Y$ as the instrument. The first stage $F$-statistic for the strength of the instrument in the IV analysis was 10, and the Kleibergen-Paap $r k$ LM $P$-value was 0.009 for all outcomes considered. There was no relationship between maternal serum ferritin and offspring BP, WC, or BMI using both methods. There was also no significant difference between the OLS and the IV analyses regression coefficients for any of the outcomes considered.

\section{Discussion}

We have examined the association of maternal iron status with adult offspring's BP, BMI, and WC. We have compared two methods; confounder-adjusted multivariable analyses, and analyses where we used a genetic mutation $(C 282 Y)$ in the $H F E$ gene as an instrumental variable for maternal iron status. We have found that maternal iron status was not associated with the three offspring outcomes considered in this study using either method. There was also no statistical evidence that the associations differed between these two statistical approaches. In our sample, maternal $C 282 Y$ genotype was associated with ferritin level. This supports our hypothesis that HFE $C 282 Y$ could be used as an instrumental variable for iron status.

There are two published studies examining the relationship of maternal iron intake and hemoglobin levels in pregnancy with children's blood pressure at 3 and 7 years of age..$^{22,23}$ However, to our knowledge, there are no previous studies examining the relationship of maternal iron status with adult offspring blood pressure and adiposity using either approach (OLS or MR). Belfort et al reported a positive, rather than an inverse, association between maternal iron intake in pregnancy and child SBP at 3 years, ${ }^{23}$ while Brion et al found no association. ${ }^{22}$ However, iron intake in pregnancy is not considered a good indicator of iron status, as this largely depends on women's iron reserves before pregnancy. 


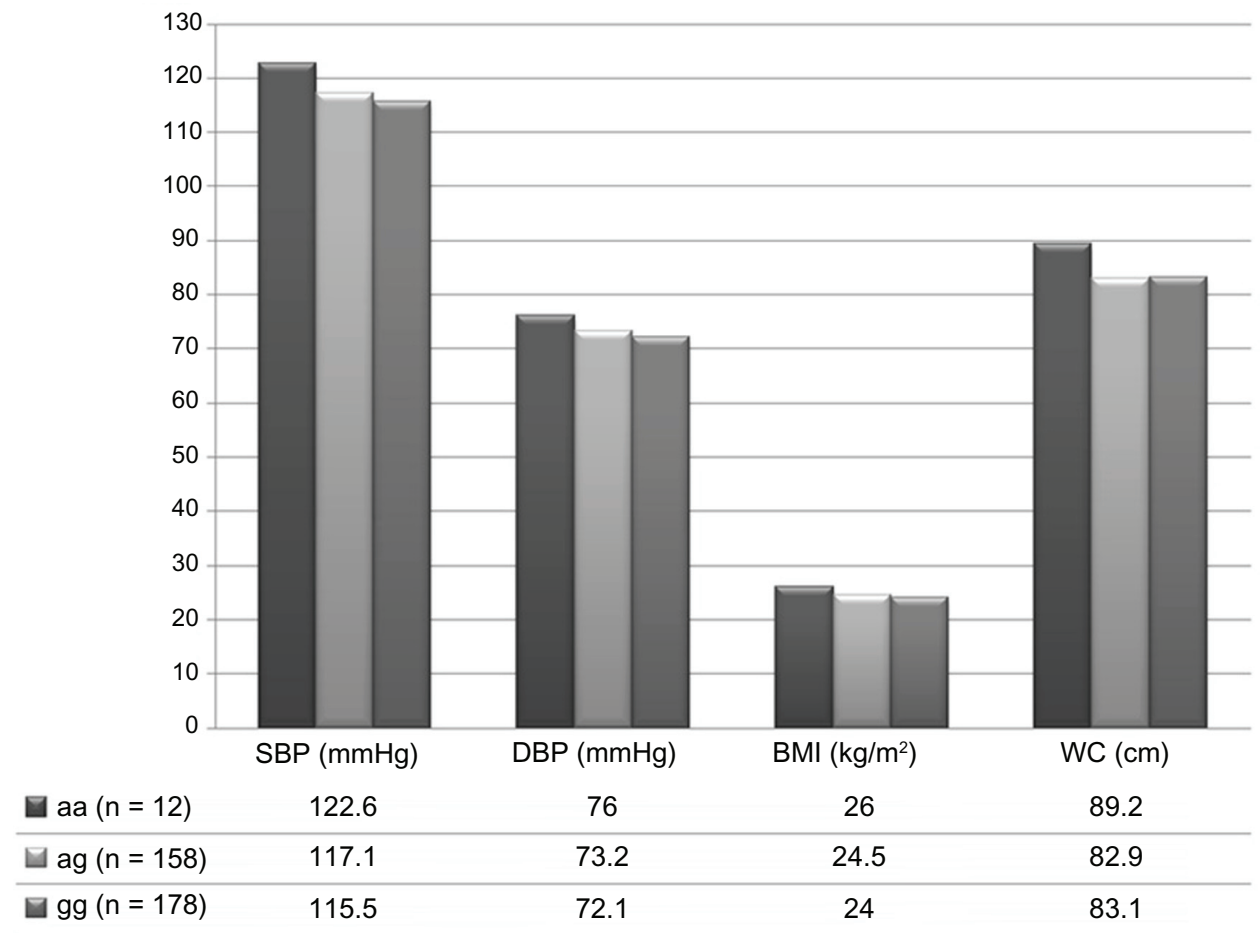

Figure I Mean offspring blood pressure (BP), body mass index (BMI), and waist circumference (WC) per maternal genotype.

Fortified foods may have the potential to reduce micronutrient deficiencies including iron during pregnancy. ${ }^{25}$ However, a study in Denmark found that $40 \%$ of women of childbearing age had low iron status despite having a national iron fortification program of flour. ${ }^{26}$

\section{Study strengths and weaknesses}

A key strength of this study is that it is the first to examine the association of maternal iron status with offspring blood pressure and adiposity in a human population. Thus, we have taken observations from previous animal work ${ }^{2-5}$ into a human population. We have used serum ferritin as a measure of iron status, as there is evidence that ferritin alone provides a good

Table 3 Univariable $P$-values of the association between maternal serum ferritin and maternal $C 282 Y$ with potential confounders in the relationship between maternal iron status and offspring outcomes

\begin{tabular}{lll}
\hline & Maternal ferritin & Maternal C282Y \\
\hline Maternal age & $<0.0001$ & 0.9 \\
Offspring age & $<0.0001$ & 0.9 \\
Offspring gender & 0.3 & 0.4 \\
Maternal vegetarian status & $<0.0001$ & 0.8 \\
Offspring vegetarian status & 0.003 & 0.9 \\
Maternal smoking & 0.1 & 0.1 \\
Maternal social class & 0.5 & 0.9 \\
Maternal BMI & 0.2 & 0.8 \\
\hline
\end{tabular}

Abbreviation: BMI, body mass index. approximation of total body iron reserves, as validated by transferrin receptor-to-ferritin ratios in people with unelevated C-reactive protein..$^{22}$ There is however, a possibility that this approximation may vary in pregnant women.

We have also tried to examine the association using two statistical approaches with different underlying assumptions for assessing causality that complement each other. The multivariable approach assumes that potential confounding factors are accounted for, correctly measured and modeled, and that there is no possibility of reverse causality. In this case, reverse causality is unlikely (ie, we cannot see how offspring outcomes could influence the mothers iron status). However, residual confounding is still possible with this approach.

We did not have information on maternal iron intake during pregnancy. We have also used maternal ferritin concentration assessed many years after they were pregnant with the offspring, and are therefore assuming in these analyses that this is a reasonable proxy for maternal iron status during pregnancy. Any measurement error is likely to be non-differential with respect to the offspring outcomes that we have assessed here, and therefore the expectation would be that results would be biased towards the null. Thus, this measurement error might explain the null multivariable observations. For the instrumental variable analyses, using maternal iron concentrations outside of pregnancy 
Table 4 Association of maternal iron status with offspring blood pressure and adiposity measures using maternal C282Y status as an instrumental variable

\begin{tabular}{|c|c|c|c|}
\hline Outcome & $\begin{array}{l}\text { OLS* mean difference } \\
\text { in outcome per } 10 \mu \mathrm{g} / \mathrm{L} \text { greater } \\
\text { maternal ferritin }(95 \% \mathrm{Cl})\end{array}$ & $\begin{array}{l}\text { IV** regression mean difference } \\
\text { in outcome per } 10 \mu \mathrm{g} / \mathrm{L} \text { greater } \\
\text { maternal ferritin }(95 \% \mathrm{Cl})\end{array}$ & $\begin{array}{l}P \text {-value*** } \\
\text { for the difference in } \\
\text { regression coefficients }\end{array}$ \\
\hline $\begin{array}{l}\text { Systolic BP } \\
\text { (SBP) (mmHg) }\end{array}$ & $0.03(-0.1$ to 0.1$)$ & $0.2(-0.2$ to 0.6$)$ & 0.5 \\
\hline $\begin{array}{l}\text { Diastolic BP } \\
\text { (DBP) }(\mathrm{mmHg})\end{array}$ & $0.04(-0.07$ to 0.2$)$ & $0.2(-0.07$ to 0.5$)$ & 0.6 \\
\hline $\begin{array}{l}\text { Body mass index } \\
\text { (BMI) }\left(\mathrm{kg} / \mathrm{m}^{2}\right)\end{array}$ & $0.02(-0.02$ to 0.07$)$ & $0.1(-0.03$ to 0.2$)$ & 0.5 \\
\hline $\begin{array}{l}\text { Waist circumference } \\
\text { (WC) }(\mathrm{cm})\end{array}$ & 0.1 (0 to 0.2$)$ & $0.07(-0.3$ to 0.5$)$ & 0.9 \\
\hline
\end{tabular}

Notes: *Multivariable ordinary least squares models adjusted for maternal age, child age, and gender in all models, and additionally for maternal vegetarian status in the DBP model, and maternal BMI and social class (NS-SEC) in the BMI and WC models; **instrumental variable two-stage least squares (2SLS), first stage F-statistic = I0; *** derived using 10,000 bootstrapped replications to estimate the standard errors of the differences between the IV and multivariable OLS regression estimates.

Abbreviations: $\mathrm{Cl}$, confidence interval; IV, instrumental variable; OLS, ordinary least squares.

is not a major limitation as the genetic variant will have resulted in subtle differences in iron status throughout the woman's lifecourse including during pregnancy. This approach is also less influenced by residual confounding. However, the instrumental variable analysis assumes that the instrument is associated with the risk factor of interest, which we know is the case from previous studies and which we have demonstrated here, though the first stage $F$-statistic of 10 suggests that the instrument's strength to identify the estimator (maternal ferritin) is borderline. ${ }^{19}$ This would bias the instrumental variable analysis towards the null results seen in the multivariable analysis. Using another measure of iron stores during pregnancy, such as transferrin receptor-to-ferritin ratio, which is not affected by inflammation, could strengthen the genetic instrument.

It is further assumed that the instrumental variable (for this study, the $C 282 Y$ mutation in the HFE gene) is not associated with any measured or unmeasured confounding, ie, it is naturally randomized in the population. This is confirmed by testing the association between maternal $C 282 Y$ and selected measured factors in our data as shown in Table 3. However, one of the conditions of IV analysis is that the instrument should not be related to the outcome except through the exposure of interest ( $\mathrm{Z}$ related to $\mathrm{Y}$ only through $\mathrm{X}$ ). The fact that maternal genotype is linked to offspring genotype may constitute a violation to this assumption. This would be the case in all transgenerational studies that attempt to use a MR design. The ideal way to tackle this problem would be to measure offspring genotype and adjust for it. One of the main limitations in this study is that offspring genotype was not measured. There is some evidence linking $C 282 Y$ heterozygotes with an increased risk of cardiovascular (CV) disease. ${ }^{27,28}$ However, other studies show no association. ${ }^{29,30}$
If there is a true positive relationship between offspring genotype and their BP, this may counteract an inverse relationship between maternal genotype (used as a proxy for maternal iron status here) and offspring's BP, potentially leading to a spurious non-association in the relationship of interest. However, it could be argued that it is the iron status at the time of gestation that is the contributing factor to $\mathrm{CV}$ risk, and not the phenotype of the fetus.

The study's response rate of $10 \%$ meant that the study had a smaller sample size than initially planned. MR studies require sample sizes larger than classical epidemiological studies due to reduced precision of the estimates as genotype usually only explains a proportion of the variation in the environmental exposure of interest. The relatively poor response rate may be due to the indirect recruitment methods used (through mothers), as well as the necessity of visiting the GP.

\section{Conclusion}

We found no association between maternal iron status and offspring's blood pressure and adiposity using both multivariable ordinary least squares regression and instrumental variable modeling with maternal $C 282 Y$ mutation in the $H F E$ gene as the instrument. However, the relatively small study sample for a MR design and the lack of measurement of the offspring $C 282 Y$ genotype in order to adjust for any possible effect on the relationship of interest are likely to push the study results towards accepting the null hypothesis. Therefore, replication of findings in studies that avoid these limitations should be considered.

\section{Author contributions}

JEC, DCG, and HJM contributed to the study design. NAA organized the data collection and processing, performed the 
statistical analysis, with assistance from DAL and DCG, and wrote the first draft of the paper. All authors have fully participated in the reporting stage and have seen and approved the final draft of the paper.

\section{Acknowledgments}

This work was supported by the Wellcome Trust [Grant number WT87789 to NAA], and the Rural Environmental Scientific and Analytical Service (RESAS) of the Scottish Government and the European Union (EARNEST) to HJM. DAL works in a centre that receives support from the UK Medical Research Council. The comments in this paper are those of the authors and not necessarily any funding body. No funders influenced the data collection, analysis, or interpretation of the results for this study.

We would like to thank all the participants in this study, Diane Threapleton and Camilla Nykjaer for data collection and database organisation, Christina Robertson for help with the mailing and recruitment, and James Thomas for help with the database.

\section{Disclosure}

The authors report no conflicts of interest in this work.

\section{References}

1. WHO. Micronutrient deficiencies; Iron deficiency anaemia. 2011. Available at: http://www.who.int/nutrition/topics/ida/en/index.html. Accessed 25 July, 2011.

2. Gambling L, Danzeisen R, Fosset C, et al. Iron and copper interactions in development and the effect on pregnancy outcome. $J$ Nutr. 2003;133(5 Suppl 1):1554S-1556S.

3. Gambling L, Dunford S, Wallace D, et al. Iron deficiency during pregnancy affects postnatal blood pressure in the rat. $J$ Physiol. 2003 552(Pt 2):603-610.

4. Crowe C, Dandekar P, Fox M, Dhingra K, Bennet L, Hanson M. The effects of anaemia on heart, placenta and body weight, and blood pressure in fetal and neonatal rats. $J$ Physiol. 1995;488(Pt 2):515-519.

5. Gambling L, Dunford S, McArdle H. Iron deficiency in the pregnant rat has differential effects on maternal and fetal copper levels. $J$ Nutr Biochem. 2004;15:366-372.

6. Gambling L, McArdle HJ. Iron, copper and fetal development. Proc Nutr Soc. 2004;63(4):553-562.

7. Lawlor DA, Davey SG, Kundu D, Bruckdorfer KR, Ebrahim S. Those confounded vitamins: what can we learn from the differences between observational versus randomised trial evidence? Lancet. 2004;363(9422):1724-1727.

8. Smith D. 'Mendelian randomization': can genetic epidemiology contribute to understanding environmental determinants of disease? Int J Epidemiol. 2003;32(1):1-22.

9. Smith GD, Lawlor DA, Harbord R, Timpson N, Day I, Ebrahim S. Clustered environments and randomized genes: a fundamental distinction between conventional and genetic epidemiology. PLoS Med. 2007;4(12):e352.

10. Lawlor DA, Harbord RM, Sterne JA, Timpson N, Davey Smith G. Mendelian randomization: using genes as instruments for making causal inferences in epidemiology. Stat Med. 2008;27(8): 1133-1163.
11. Rhodes D, Raha-Chowdhury R, Cox T, Trowsdale J. Homozygosity for the predominant Cys282Tyr mutation and absence of disease expression in hereditary haemochromatosis. J Med Genet. 1997;34:761-764.

12. Willis G, Wimperis J, Smith K, Fellows I, Jennings B. Haemochromatosis gene C282Y homozygotes in an elderly male population. Lancet. 1999; 354:221-222.

13. Chan AT, Ma J, Tranah GJ, et al. Hemochromatosis gene mutations, body iron stores, dietary iron, and risk of colorectal adenoma in women. J Natl Cancer Inst. 2005;97(12):917-926.

14. Cade JE, Moreton JA, O'Hara B, et al. Diet and genetic factors associated with iron status in middle-aged women. Am J Clin Nutr. 2005;82(4):813-820.

15. Beutler E, Felitti VJ, Koziol JA, Ho NJ, Gelbart T. Penetrance of 845G--> A (C282Y) HFE hereditary haemochromatosis mutation in the USA. Lancet. 2002;359(9302):211-218.

16. Jackson H, Carter K, Darke C, et al. HFE mutations, iron deficiency and overload in 10,500 blood donors. Br J Haematol. 2001;114(2):474- 484.

17. Zimmermann MB. Methods to assess iron and iodine status. Br J Nutr. 2008;99 Suppl 3:S2-S9.

18. Cade JE, Burley VJ, Greenwood DC; UK Women's Cohort Study Steering Group. The UK Women's Cohort Study: comparison of vegetarians, fish-eaters and meat-eaters. Public Health Nutr. 2004;7(7): 871-878.

19. Stock J, Staiger D. Instrumental variables regression with weak instruments. Econometrica. 1997;65(3):393-415.

20. Baum CF, Schaffer ME, Stillman S. Enhanced routines for instrumental variables/generalized method of moments estimation and testing. Stata Journal. 2007;7(4):465-506.

21. Whitworth JA; World Health Organization, International Society of Hypertension Writing Group. 2003 World Health Organization (WHO)/ International Society of Hypertension (ISH) statement on management of hypertension. J Hypertens. 2003;21(11):1983-1992.

22. Brion MJ, Leary SD, Smith GD, McArdle HJ, Ness AR. Maternal anemia, iron intake in pregnancy, and offspring blood pressure in the Avon Longitudinal Study of Parents and Children. Am J Clin Nutr. 2008;88:1126-1133.

23. Belfort MB, Rifas-Shiman SL, Rich-Edwards JW, Kleinman KP, Oken E, Gillman MW. Maternal iron intake and iron status during pregnancy and child blood pressure at age 3 years. Int J Epidemiol. 2008;37(2): 301-308.

24. Yang Z, Dewey KG, Lönnerdal B, et al. Comparison of plasma ferritin concentration with the ratio of plasma transferrin receptor to ferritin in estimating body iron stores: results of 4 intervention trials. Am J Clin Nutr. 2008;87(6):1892-1898.

25. Yang Z, Huffman SL. Review of fortified food and beverage products for pregnant and lactating women and their impact on nutritional status. Matern Child Nutr. 2011;7 Suppl 3:19-43.

26. Milman N, Byg KE, Ovesen L, Kirchhoff M, Jürgensen KSL. Iron status in Danish women, 1984-1994: a cohort comparison of changes in iron stores and the prevalence of iron deficiency and iron overload. Eur J Haematol. 2003;71(1):51-61.

27. Tuomainen TP, Kontula K, Nyyssönen K, Lakka TA, Heliö T, Salonen JT. Increased risk of acute myocardial infarction in carriers of the hemochromatosis gene Cys282Tyr mutation: a prospective cohort study in men in eastern Finland. Circulation. 1999;100(12):1274-1279.

28. Rasmussen ML, Folsom AR, Catellier DJ, Tsai MY, Garg U, Eckfeldt JH. A prospective study of coronary heart disease and the hemochromatosis gene (HFE) C282Y mutation: the Atherosclerosis Risk in Communities (ARIC) study. Atherosclerosis. 2001;154(3):739-746.

29. Gunn IR, Maxwell FK, Gaffney D, McMahon AD, Packard CJ. Haemochromatosis gene mutations and risk of coronary heart disease: a west of Scotland coronary prevention study (WOSCOPS) substudy. Heart. 2004;90(3):304-306.

30. Ellervik C, Tybjærg-Hansen A, Grande P, Appleyard M, Nordestgaard BG. Hereditary hemochromatosis and risk of ischemic heart disease: a prospective study and a case-control study. Circulation. 2005;112(2): 185-193. 


\section{Publish your work in this journal}

Clinical Epidemiology is an international, peer-reviewed, open access journal focusing on disease and drug epidemiology, identification of risk factors and screening procedures to develop optimal preventative initiatives and programs. Specific topics include: diagnosis, prognosis, treatment, screening, prevention, risk factor modification, systematic

Submit your manuscript here: http://www.dovepress.com/clinical-epidemiology-journal reviews, risk \& safety of medical interventions, epidemiology \& biostatical methods, evaluation of guidelines, translational medicine, health policies \& economic evaluations. The manuscript management system is completely online and includes a very quick and fair peer-review system, which is all easy to use. 